Rev. Biol, Trop., 9(1):17-21, 1961

\title{
Investigaciones sobre Dermatitis Esquistosómica en el Lago de Pátzcuaro, Michoacán, México
}

\author{
I. Descripción de una nueva forma de cercaria*
}

por

Eduardo Caballero y C.**

Luis Flores-Barroeta**

y

E. Hidalgo E.**

(Recibido para su publicación el 22 de noviembre de 1960)

En varias regiones de la República Mexicana, tales como Chupícuaro en el Lago de Pátzcuaro, Michoacán, y la Zona del Río Mante y sus afluentes, en el Estado de Tamaulipas, se presentan en el hombre pequeñas pápulas en las piernas, características de la dermatitis producida por la penetración de furcocercarias de tremátodos esquistosómidos de aves acuáticas migratorias, de los géneros Ornithobilharzia Odhner, 1912, Trichobilharzia Skrjabin \& Zharkow, 1920, y otros.

Para precisar estos hechos, hemos realizado exploraciones de las playas del balneario de Chupícuaro, en setiembre de 1957, y en febrero de 1959 de los canales de los embarcaderos, orillas e islas del Lago de Pátzcuaro.

En la primera ocasión recogimos de las piedras cerca de la playa y bajo el agua, y de raíces de Eichbornia speciosa Kunth, ejemplares de Physa mexicana var. tolucensis, Helisoma tenuis var. boucardi, Sphaerium triangularis Say y S. subtransversum Prime. De ninguno de ellos se obtuvo salida de cercarias al colocar los moluscos bajo observación constante en agua del mismo lago.

En la segunda expedición obtuvimos numerosos ejemplares de Physa mexicana tolucensis en los canales 1 y 2 del embarcadero y adheridos a hojas de

* Trabajo leído en el Tercer Congreso Nacional de la Asociación Mexicana de Microbiología, celebrado en la Ciudad de México, D. F., en octubre de 1960.

Estas investigaciones se han realizado gracias a la ayuda económica brindada por el Instituto Nacional de la Investigación Científica de México.

* Laboratorio de Helmintología, Escuela Nacional de Ciencias Biológicas, Instituto Po. litécnico Nacional, México, D. F. 
Nymphaea mexicana Zucc. y entre raíces de Eichbornia speciosa Kunth., así como de las piedras de la isla de Tecuenita, cerca de las de Tecuena y Pacanda, atrás de Janitzio; en los talluelos de tule, Scirpus sp., en las orillas de la isla de Ihuatzio y en la playa de la isla Tecuenita, encontramos numerosos ejemplares de Helisoma tenui, boucardi; y de la misma localidad en la isla de Ihuatzio y de raíces de lirio acuático (Eichbornia) de la zona próxima al canal 2 en el embarcadero del lago, ejemplares de Sphaerium triangularis Say.

Estas últimas dos especies dieron nuevamente resultados negativos. No así los ejemplares de Physa mexicana tolucensis. Estos fueron colocados al capturarlos en grupos de 2 ó 3 en pequeños frascos de cristal, y observados cada 15 minutos durante el recorrido del lago. Desde la primera observación se les vió liberar gran cantidad de cercarias.

Al llegar a la estación limnológica, se separaron los moluscos, cambiándoles el agua; se notó inmediatamente nueva salida de buen número de cercarias, que permanecieron en el líquido alrededor de 24 horas. Al cabo de este tiempo se cambió agua por tercera vez y las cercarias emergieron nuevamente, permaneciendo vivas otras 24 horas; todavía se repitió esta operación una cuarta vez con los mismos resultados. Las cercarias fueron examinadas y estudiadas todos los días de su salida, entre las 11 a. m. y las 5 p. m.; posteriormente se fijaron añadiendo al agua líquido de Railliet y Henry.

Las cercarias obtenidas en esta ocasión corresponden a una especie nueva, que describimos a continuación.

\section{Cercaria patzcuarensis n. sp.}

Los ejemplares miden $0,507-0,522 \mathrm{~mm}$ de longitud total; la parte correspondiente al cuerpo es oblonga, más ancha en su parte posterior, con una región convexa y otra cóncava y tiene 0,179 - 0,179 mm de largo por 0,104 - 0,104 mm de ancho; la extremidad caudal es gruesa y robusta, más gruesa en su parte anterior que en la distal y alcanza 0,373 - 0,402 $\mathrm{mm}$ de longitud por $0,030-0,037 \mathrm{~mm}$ de anchura. La relación entre la longitud de la cola a la del cuerpo es 1:2,08; la cola está revestida por una epidermis de tipo empalizada en la que hay numerosos cuerpecitos esféricos y pequeños a manera de protuberancias. En el cuerpo y en posición subterminal se halla una ventosa muscular que mide $0,042-0,042 \mathrm{~mm}$ de largo y $0,042-0,046 \mathrm{~mm}$ de ancho; el acetabulum es ligeramente mayor que la ventosa anterior, está situado por detrás del ecuador del cuerpo y tiene 0,042 - 0,042 mm de longitud por 0,046 - 0,050 mm de anchura. La relación entre el tamaño de las dos ventosas es 1:1 x $1: 1,09$ a $1: 1,09 \times 1: 1,19$. El aparato digestivo consta de una amplia boca en posición terminal, de una corta prefaringe y de una faringe ovoidea, de paredes fuertemente musculosas, pequeña, que mide 0,025 - 0,046 mm de largo y 0,008 - 0,017 $\mathrm{mm}$ de ancho; a continuación hay un corto esófago, el que se resuelve en dos ciegos intestinales cortos que abrazan en parte anterior al acetabulum.

Existe una masa de células reproductoras entre el nivel del borde posterior del acetábulum y la vesícula excretora. El aparato excretor está formado por 
una amplia vesícula, de eje transversal mayor que el antero-posterior, la cual está situada por delante de la inserción de la cauda; de ella parten hacia adelante dos conductos colectores muy sinuosos, de posición lateral, que se extienden hasta el borde anterior de la faringe.

El cuerpo de la larva se distingue por ser oscuro y estar provisto de dos grandes manchas ópticas en la porción anterolateral y que son muy notables en vivo.

Estos estados larvarios al abandonar al molusco, se mueven activamente por contracciones de la cola, unas veces describiendo una línea recta y otras formando un ángulo recto entre el cuerpo y la cauda o bien ángulos agudos u obtusos; se desplazan fundamentalmente de arriba abajo, en el seno del agua contenida en los frascos y muy pocas veces se aproximan a las paredes; esta actividad la presentan durante un tiempo comprendido entre 8 y 24 horas.

\section{DISCUSION}

Las dos exploraciones realizada's en el lago de Pátzcuaro, para investigar furcocercarias causantes de dermatitis esquistosómica humana, no han puesto por e! momento de manifiesto ninguna cercaria de esta clase (1). Pero, en cambio, se ha encontrado que Physa mexicana Philippi var. tolucensis está parasitada con las formas larvarias de un tremátodo cuya cercaria ha sido designada como Cercaria patzcuarensis n. sp., estado larvario que pertenece a las cercarias dístomas, leptocercas y gimnocéfalas (2). Esta larva se diferencia de otras formas leptocercas, entre ellas la de Fasciola bepatica $L$., en primer lugar por la relación de los tamaños entre el cuerpo y la cauda; en segundo por la presencia de manchas ocelares que son muy notables en vivo; en tercero por presentar una cutícula espinosa y por último por la configuración de los aparatos excretor y digestivo (4).

\section{AGRADECIMIENTO}

Agradecemos atentamente al Sr. Biólogo Aurelio Solórzano, director de la Estación Limnológica de Pátzcuaro, la gentileza de poner a nuestra disposición una lancha de motor que usamos en las recolecciones de 1959.

\section{RESUMEN}

Dos series de investigaciones realizadas para comprobar la presencia de dermatitis esquistosómica en el Lago de Pátzcuaro, Michoacán, han dado resultados negativos en las siguientes especies de moluscos: Physa mexicana tolucensis, Helisoma tenuis boucardi, Spbaerium triangularis y S. subtransversum. De ejemplares de Pbysa mexicana tolucensis de la isla Tecuenita, se obtuvieron abundantes cercarias de una especie nueva, que se describe bajo el nombre de Cercaria patzcuarensis. 


\section{SUMMARY}

Two series of collections in Lake Patzcuaro, Michoacan, Mexico, made with the purpose of investigating the presence of schistosome - dermatitis (swimmer's itch) have yielded negative results in the snails collected +- Physa mexicana tolucensis, Helisoma tenuis boucardi, Spbaerium triangularis and S. subtransversum. A new distome, leptocercous, gymnocephalous cercaria was obtained from Prysa mexicana tolucensis, and described here as Cercaria patzcuarensis n. sp.

\section{REFERENCIAS}

1. CORT, 'W. W.

1915. Some North American larval trematodes. Ill. Biol. Monogr., 1(4): 447 - 532.

2. DaWES, B.

1949. The Trematoda, with special reference to British and other European forms. 644 pp. Cambridge University Press. England.

3. Dubors, G.

1929. Les cercaires de la region de Neuchatel. Thèse, Univ. Neuchatel, 177 pp.

4. Wesenberg-Lund, C.

1934. Contributions to the development of the Trematoda Digenea Part. II. The biology of the fresh water cercariae in Danish freshwaters. Mem. Acad. Sci. Lettr. Danemark, Sec. Sci., 9 ser., 5(3): 1-223. 


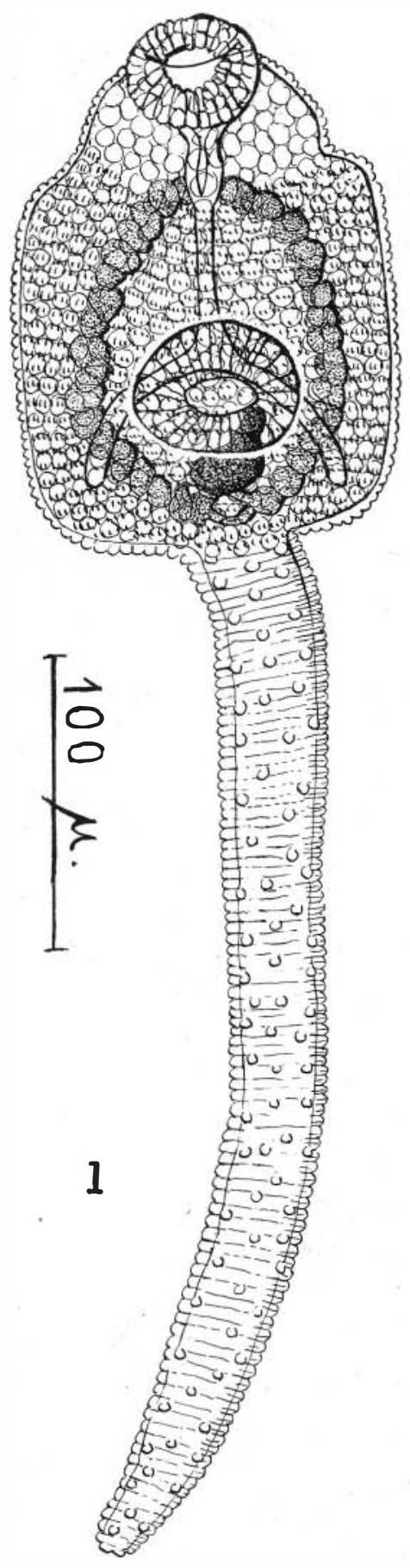

